\title{
Diseño de materiales para el aprendizaje de habilidades jurídicas fundamentales
}

\author{
José García Añón \\ Institut Universitari de Drets Humans. Profesor del Departament \\ de Filosofia del Dret Moral i Política de la Universitat de València \\ garciaj@uv.es
}

\section{Ricardo Juan Sánchez}

Profesor del Departament de Dret Administratiu i Processal de la Universitat de València

ricardo.juan@uv.es

\section{Roberto Pérez Salom}

Institut Universitari de Drets Humans. Profesor del Departament de Dret Internacional de la Universitat de València

jose.r.perez@uv.es

\section{Ángeles Solanes Corella}

Institut Universitari de Drets Humans. Profesora del

Departament de Filosofia del Dret Moral i Política de la

Universitat de València | angeles.solanes@uv.es

Francisco González Castilla

Profesor del Departament de Dret Mercantil de la

Universitat de València

francisco.gonzalez@uv.es

\section{Jorge Correa Ballester}

Profesor del Departament de Dret Financer i Història del Dret de la Universitat de València

jorge.correa@uv.es

\section{Mario Clemente Meoro}

Departament de Dret Civil de la Universitat de València mario.clemente@uv.es

\section{María José Añón Roig}

Institut Universitari de Drets Humans. Profesora del Departament de Filosofia del Dret Moral i Política de la Universitat de València | mariaj@uv.es

Vicenta Cervelló Donderis

Profesora del Departament de Dret Penal de la

Universitat de València

vicenta.cervello@uv.es

Marta Lalaguna Holzwarth

Becaria de colaboración de la Universitat de València. Curso $2007 / 2008$

|Fecha presentación: 05/06/2008 | Aceptación: 16/09/2008 |Publicación: 22/12/2008

\begin{abstract}
Resumen
Se describe el proceso de generación de materiales cuyo contenido son las denominadas habilidades jurídicas fundamentales. Se han elaborado con la herramienta de materiales en línea de la plataforma docente de la Universitat de València, Aula Virtual (http://aulavirtual.uv.es) y en la web (www.uv.es/legalskills). Se han utilizado diversas aplicaciones informáticas: eXelearning para empaquetar en formato SCORM, programas de simulación, edición de video... Los videos se han depositado en un servidor streaming en formato flash y mp4. Los materiales pretenden favorecer el aprendizaje autónomo de los estudiantes de Primer Ciclo de la Licenciatura en Derecho y paliar las deficiencias en la adquisición coherente de estas habilidades.
\end{abstract}

Palabras clave: habilidades jurídicas fundamentales, plataforma docente virtual, scorm, eXelearning, video

\section{Resum}

Es descriu el procés de generació de materials el contingut dels quals són les denominades habilitats jurídiques fonamentals. S'han elaborat amb l'eina de materials en línia de la plataforma docent de la Universitat de València, Aula Virtual (http://aulavirtual.uv.es) i en una pàgina web (www.uv.es/legalskills). S'han utilitzat diverses aplicacions informàtiques: eXelearning per a empaquetar en format SCORM, programes de simulació, edició de video... Els videos s'han dipositat en un servidor streaming en format flash i mp4. Els materials pretenen afavorir l'aprenentatge autònom dels estudiants de Primer Cicle de la Llicenciatura en Dret i pal-liar les deficiències en l'adquisició coherent d'aquestes habilitats.

Paraules clau: habilitats jurídiques fonamentals, plataforma docent virtual, scorm, eXelearning, video 


\begin{abstract}
It describes the process of generating materials whose contents are the "fundamental legal skills". They have been developed through online materials in the teaching platform of the University of Valencia, (http://aulavirtual.uv.es) and on the web (www.uv.es/legalskills). We used various software applications: eXelearning to pack into SCORM format, simulation programs, video editing ... The videos have been placed on a server in streaming flash format and mp4. The materials are intended to encourage independent learning for students of Law degree and correct deficiencies in the acquisition of these skills.
\end{abstract}

Keywords: fundamental legal skills, online teaching platform, scorm, eXelearning, video

\section{Introducción}

El proyecto pretende generar materiales que favorezcan el aprendizaje autónomo de los estudiantes de Primer Ciclo de la Licenciatura en Derecho a través de las denominadas habilidades jurídicas fundamentales por medio de la utilización de la plataforma docente de la Universitat de València, Aula Virtual (http://aulavirtual.uv.es).

Los materiales que estamos desarrollando se sitúan en el contexto de las competencias o habilidades fundamentales (Fundamental Skills) aplicadas al ámbito del Derecho (Basic Legal Skills). Las competencias se pueden definir como "una combinación dinámica de atributos - con respecto al conocimiento y su aplicación, a las actitudes y a las responsabilidades- que describen los resultados del aprendizaje de un determinado programa, o cómo los estudiantes serán capaces de desenvolverse al finalizar el proceso educativo." (González y Wagenaar 2003: 280; García Martínez 2007: 163 y ss.; León Benítez, 2007: 179 y ss). Se trata, en definitiva, de la capacidad de poder tratar con las personas y sus problemas como un jurista competente, ético y socialmente responsable (Maughan y Webb 2005).

Hay una amplia tradición de estructurar la enseñanza de las habilidades jurídicas fundamentales en los planes de estudio del ámbito anglosajón (véase, por ejemplo, sin ánimo de ser exhaustivos: Lasswell y McDougal 1943; 1999: 90 y ss.; Gold, Makie y Twining 1988; Webb y Maughan 1996; o Williams 2002). De hecho, aparecen en el primer informe del Comité de Asesoramiento sobre Educación y Conducta Jurídica (ACLEC 1996), no tan solo como requisito para que los estudiantes reciban una educación adecuada, sino también para que su formación se corresponda con las demandas de la profesión, en ocasiones, cambiantes ${ }^{1}$. Por este motivo, la Quality Assurance Agency del Reino Unido ha definido a través de las Law Benchmark Statements las habilidades que pueden esperarse de un graduado en Derecho en términos de habilidades y destrezas necesarias para desarrollar con competencia esa materia. Una primera versión fue aprobada en 2000 y revisada en 2007. Lo interesante de esta aproximación, además de delimitar las habilidades requeridas, en distintos niveles, es la determinación y concreción de los elementos que sirven para graduar una evaluación de las mismas².
Esto supone también adoptar una nueva perspectiva en la que la determinación y el desarrollo de las competencias se convierte en el eje del diseño y planificación curricular, así como también de las actividades y dinámicas que se desarrollan (Miguel 2006). De esta manera se modifican las formas de aprendizaje y enseñanza, por lo que no se pretende ni evalúa la acumulación de conocimientos, sino las capacidades de los individuos en determinados contextos de aprendizaje.

En nuestro ámbito, también se ha insistido en la determinación de las competencias y habilidades en diversos documentos e informes como el Tuning (González y Wagenaar 2003) o el Libro Blanco de Derecho (ANECA 2006: 89 y ss.), entre otros, aunque la propuesta y clasificación de las competencias, a veces, resulta dispar3.

En nuestro caso, como se verá más adelante, hemos optado por desarrollar en estos materiales aquellas competencias y habilidades que, teniendo un carácter transversal o genérico, requieren ciertas peculiaridades en el ámbito jurídico. Hemos utilizado y adaptado las propuestas del profesor Jesús Morales Arrizabalaga, de la Universidad de Zaragoza, y fundador en 1997 de la lista de distribución PAIDEIUS sobre innovación didáctica del Derecho (http://www.rediris.es/list/info/paideius.html) quien, desde hace varios años, imparte la asignatura de habilidades jurídicas como de libre configuración. Esto es, entendemos que las competencias genéricas y las específicas "deben ser entrenadas" conjuntamente (García Martínez 2007: 172). En concreto, y por sintetizar, hemos dividido los contenidos en:

a) introducción y conceptos;

b) información jurídica;

c) lectura y comprensión del Derecho;

d) comunicación escrita y comunicación oral;

e) valores y estudio del Derecho;

f) la profesión de jurista.

¿Qué pretendemos? Por una parte generar unos materiales cuyos contenidos y procesos puedan desarrollar el aprendizaje de habilidades jurídicas y, por otra parte, recopilar, sistematizar y permitir el fácil acceso de material audiovisual a través de la plataforma docente de la Universitat de València, hasta que exista un repositorio de contenidos

\footnotetext{
${ }^{1}$ Las habilidades que recomienda el informe son: "i. the construction of logical argument; ii. the capacity for abstract manipulation of complex ideas; iii. the systematic management of complex factual information; iv. intelligent, critical reading of texts; $v$, the use of the English language at all times with scrupulous care and integrity; vi. the related ability to communicate orally and in writing in a clear, consistent and compelling way; vii.competence in retrieving, assessing and using legal texts and information including information technology skills."

2 Las habilidades que se describen son: knowledge, application and problem-solving; sources and research; analysis, synthesis, critical judgement and evaIuation; autonomy and ability to learn; communication and literacy; numeracy; information technology; working in teams or groups.(http://www.qaa.ac.uk/academicinfrastructure/benchmark/statements/Law07.pdf). Hay que recordar que las habilidades clásicas o DRAIN son las que giran en torno a: drafting, research, advocacy, interviewing and negotiation.

3 Puede verse una descripción y comparación de las propuestas de competencias que se deben aprender en el grado de Derecho en Delgado García (2005: 18-32). Una propuesta reciente de articulación de competencias se encuentra en Dasí et al. (2007: 51 y ss).
} 


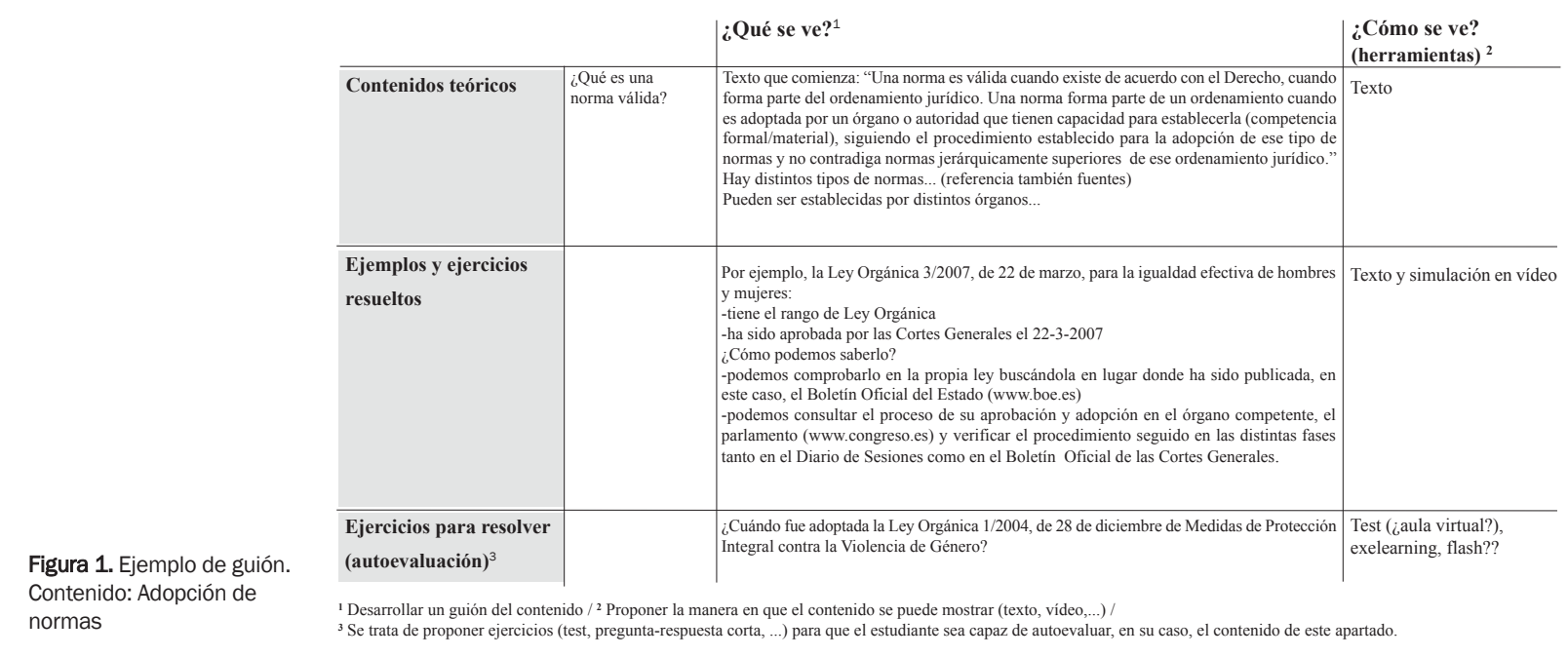

que permita realizar una búsqueda de estos materiales. Los contenidos elaborados podrían servir como punto de referencia para una asignatura similar a la existente en otros países, que incluso se ha incorporado en planes de estudio actualmente en proceso de reforma ${ }^{4}$. En su caso, como se pretende en la actualidad, puede ser un material de apoyo a profesores y estudiantes de los primeros cursos en la medida en que los contenidos son transversales a las asignaturas del grado de Derecho ${ }^{5}$. Posteriormente, la estructura puede mejorarse, en relación a la oferta de diversos niveles de complejidad y especialidad ${ }^{6}$.

Por tanto, el proyecto de generación de nuevos materiales recoge las siguientes características: a) potenciación del aprendizaje del estudiante a través de la incorporación y experimentación con nuevas metodologías, que favorece su mayor implicación y autonomía; b) importancia de los objetivos competenciales en el diseño de los contenidos del programa; c) transversalidad de conocimientos. Los materiales podrán ser utilizados de forma complementaria como herramientas de formación en los cursos de Primer Ciclo de la actual licenciatura en Derecho, al igual que en la materia común de Introducción al Derecho de los futuros planes de estudio y d) lo anterior exige coordinación del equipo de profesores tanto en el diseño y coherencia de los contenidos como en la estructuración de las dinámicas.

Se trata de potenciar y fortalecer una serie de contenidos y habilidades trasversales y básicas que afectan a las materias jurídicas de Primer Ciclo y que pueden ser desarrolladas de forma complementaria con el trabajo autónomo de los estudiantes. En los materiales, junto a la exposición de contenidos se mostrará cómo realizar determinadas actividades que permitan entender conceptos jurídicos, buscar información jurídica, analizarla y gestionarla.

En definitiva, los objetivos del proyecto son: diseñar materiales que favorezcan el aprendizaje autónomo de los estudiantes de Primer Ciclo de la Licenciatura en Derecho; favorecer la adquisición de conocimientos jurídicos básicos de los estudiantes que llegan a la universidad a través de las denominadas habilidades jurídicas fundamentales; facilitar la uniformidad en los contenidos y habilidades mínimas que deben tener los estudiantes en los primeros cursos; avanzar en la utilización de metodologías docentes activas y de colaboración entre equipos de docentes y potenciar la utilización de las tecnologías de la información y el conocimiento en el proceso de aprendizaje.

\section{Metodología}

El proyecto tiene un carácter interdisciplinar y por ello ha contado con la colaboración de profesores de diversas áreas de conocimiento: Filosofía del Derecho, Derecho Civil, Derecho Procesal, Historia del Derecho, Derecho Internacional Público, Derecho Penal y Derecho Mercantil. Además, está previsto que en el futuro se sumen otras. Por otra parte, colaboran dos becarias en formación adscritas al proyecto y personal del Servei d'Informàtica de la Universitat.

El personal del Servei d'Informàtica de la Universitat de València ha colaborado en el asesoramiento de la integración de los materiales en la plataforma docente de la Universitat y en la instalación del Servidor Multimedia en formato streaming de manera integrada con Aula Virtual7.

Los profesores han realizado las siguientes actividades:

\footnotetext{
4 Por ejemplo, en el proyecto de Plan de Estudios de Grado de Derecho de la Universidad Carlos III de Madrid se encuentra la asignatura Destrezas y habilidades ( 6 créditos) que es de Formación Básica compartida con otras ramas de la Universidad.

5 En este caso, la no existencia de una asignatura y la transversalidad de contenidos, exige estructuras organizativas de coordinación en el curso y la titulación que permitan la planificación de las actividades, la distribución de las competencias en función del perfil profesional y la evaluación de las mismas.

${ }^{6}$ La idea no es nueva, en los años cuarenta Lasswell y McDougal propusieron crear "El libro de las destrezas" en las facultades de Derecho norteamericanas. Lo explicaban así dentro de lo que denominaban "destrezas de razonamiento": "En nuestras propuestas de reforma del plan de estudios no recomendamos cursos específicos sobre pensamiento y lenguaje, sino la preparación de materiales que puedan estar disponibles rápidamente para el estudiante de derecho durante todos los años de su entrenamiento. El incentivo para dominar los instrumentos del lenguaje y el pensamiento lo debe reforzar continuamente un cultivo de la toma de conciencia en el uso de las palabras por los profesores en el aula. Como guía y acompañamiento de varias de las destrezas valiosas para el estudiante de derecho, es aconsejable preparar y mantener actualizada, a través de revisión constante, una colección de materiales que podríamos Ilamar "El libro de las destrezas". Este libro debería contener copias, artículos originales escritos por expertos, e investigaciones aún no publicadas, y brindaría información biográfica de autores.” (Lasswell y McDougal (1943), 1999: 98-99)
} 
Figura 2. Imagen de Aula Virtual en el que aparecen todas las asignaturas en las que el alumno está matriculado, incluida "Habilitats jurídiques"
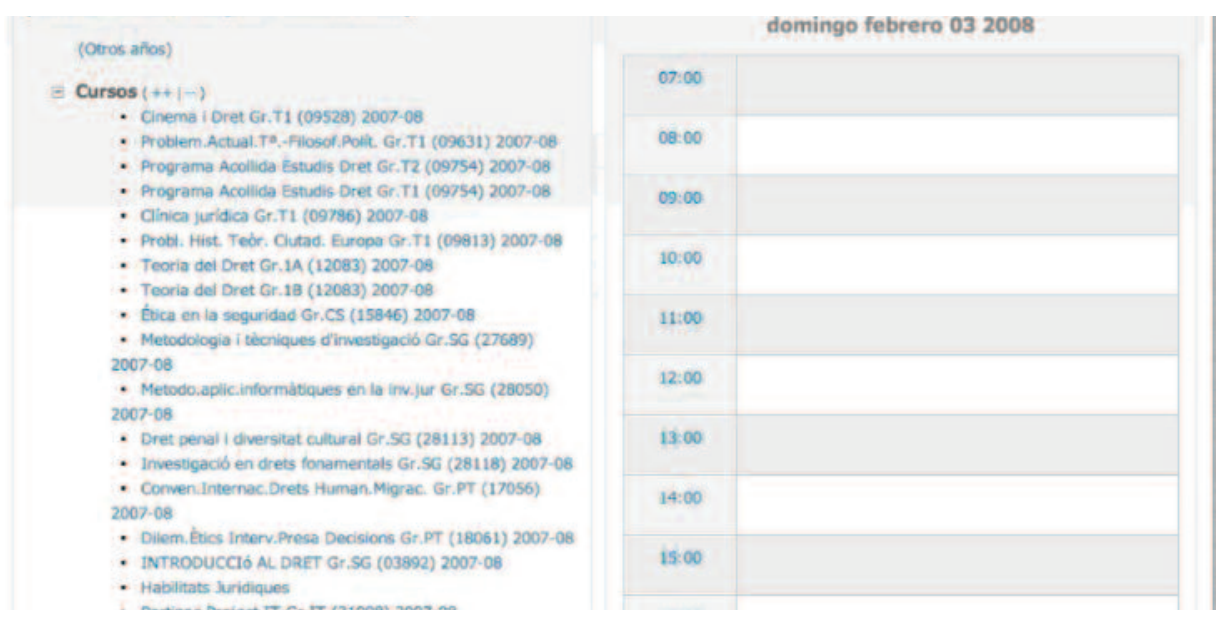

a) elaboración y desarrollo de un guión de contenidos a partir del programa de contenidos propuesto;

b) asesoramiento en los contenidos;

c) diseño y elaboración de los materiales;

d) implantación y experiencia de utilización de los materiales y

e) evaluación del uso y la calidad de los materiales a través de encuestas a estudiantes y profesores.

¿Cómo se muestran los contenidos? Las unidades didácticas. Los contenidos se muestran en unidades didácticas. Los profesores, junto con el personal de apoyo y el asesoramiento de los técnicos del Servei d'Informàtica han diseñado y elaborado los materiales. De cada unidad se ha diseñado un guión de contenidos, habilidades, y destrezas comunes. Los contenidos tienen distintos niveles y cada nivel estará recomendado para un curso determinado o, en su caso, se establecerá el grado de dificultad: iniciación, medio o avanzado.

Cada unidad didáctica incluye contenidos teóricos, ejemplos y ejercicios resueltos, ejercicios para resolver (autoevaluación) y para saber másPara saber más (bibliografía, enlaces a páginas web... véase la Figura 1).

Cada unidad se organiza de manera que se pretende conseguir que el estudiante: a) acceda a unos contenidos e información mínima del tema; b) sea capaz de aprender y experimentar a través de los ejercicios y actividades; c) pueda ampliar los conocimientos a partir de la información suplementaria que se le proporciona; y d) reflexione y, llegado el caso, discuta.

El aprendizaje se consolida con la repetición de actividades y ejercicios en los que, en un proceso de reflexión y de retroalimentación, el estudiante observa y experimenta lo que sabe, lo que hace bien o mal, y se autocorrige. De alguna manera, esto también supone una responsabilidad en lo que se aprende.

Las actividades que se plantean exigen una postura activa por parte del estudiante debido a que aprende haciendo.

Los materiales tienen diversos formatos: audiovisuales, simulaciones, realización de ejercicios interactivos/autoevaluación.

¿Dónde se pueden encontrar los contenidos? Teníamos dos posibilidades de uso de plataforma para mostrar los contenidos: la primera, a través de una página Web (http://www.uv.es/legalskills) y la segunda a través del Aula Virtual, de su herramienta Materiales en línea, en un módulo creado denominado Habilidades jurídicas. Aunque se ha desarrollado esta segunda, también se ha experimentado en la primera por las limitaciones que presenta Aula Virtual. Aún así, esta segunda opción unifica y es coherente con el resto de módulos y asignaturas de un estudiante y permite un mejor seguimiento; además, el lenguaje SCORM es usable en otras plataformas, en el caso que la Universitat de València optase por abandonar el lenguaje dotLearn.

De esta manera, cuando un estudiante entra en las asignaturas en las que se ha matriculado, también encuentra el módulo de Habilidades jurídicas fundamentales. (Figura 2)

¿Cuál es el programa de contenidos? El programa planteado recoge la estructura básica de contenidos de esta materia: Saber y Saber hacer (destrezas, competencias y conocimientos que debe tener cualquier jurista, algunos compartidos con otras materias); y Saber estar (valores de la profesión...). Las unidades didácticas se han desarrollado a partir de la siguiente estructura básica:

A. Introducción y conceptos

A.1. Historia y cultura jurídica

A.1.1. Historia y cultura

A.1.2. Terminología jurídica

A.2. Validez

.2.1. Adopción de normas

A.2.2. Publicación

A.2.3. Entrada en vigor

A.2.4. Derogación

A.2.5. Normas transitorias

A.3. La estructura de las disposiciones normativas

A.4. Las partes del Ordenamiento Jurídico

A.5.El Derecho como proceso

A.5.1. Organización de la Administración de Justicia

A.5.2.Derechos de los ciudadanos frente a la Administración de Justicia

A.5.3. La estructura de un proceso judicial

A.6. Interpretación y aplicación del Derecho

A.7. Fuentes del Derecho

\footnotetext{
7 Salvador Roca Marquina, Técnico de Sistemas; Darío Roig García, Analista Programador; José Agustín López Bueno, Analista Programador; Sergio Cubero, Analista Programador.

8 eXeLearning es un programa creado por la Auckland University of Technology y la Tairawhiti Polytechnic, financiado por el Tertiary Education Commission de Nueva Zelanda.
} 
Figura 3. Ejemplo de utilización del programa eXelearning en el diseño de ejercicios de autocorrección

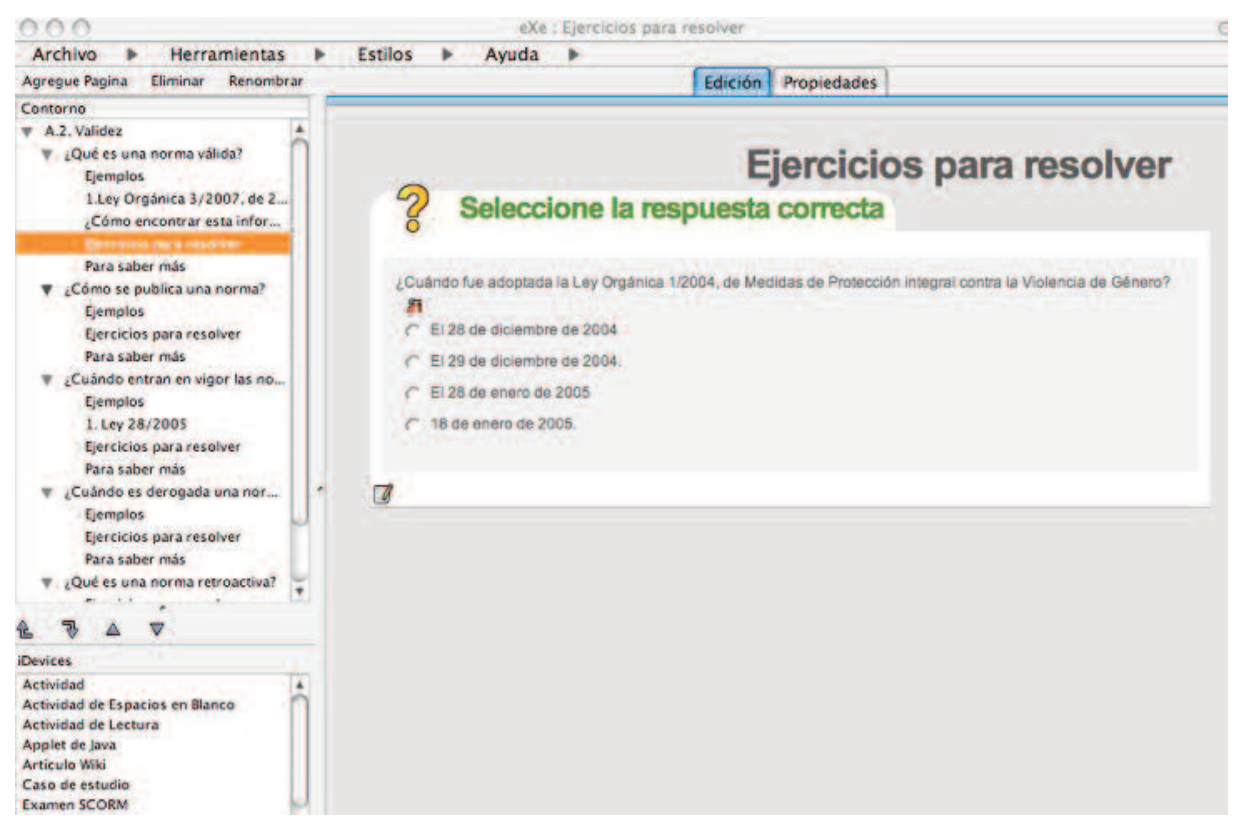

\section{B. Información jurídica}

B.1. El Derecho como objeto de conocimiento. Fuentes de información jurídica (normativas y no normativas).

B.2. Centros de documentación jurídica. La organización de la información jurídica

B.3. Edición jurídica: La edición de normas. La edición de jurisprudencia. La edición de doctrina. La edi ción de otros materiales

B.4. Búsqueda de información jurídica: normas, juris prudencia, doctrina. Bases de datos

B.5. La gestión de la información

C. Lectura y comprensión del Derecho

C.1. El lenguaje jurídico

C.2. Lectura de géneros fundamentales: manual, artí culo, monografía

D. Comunicación escrita y comunicación oral

D.1. Escritura de géneros básicos: recensión, trabajo de curso D.1.1. Elección del tema

D.1.2. Esquema

D.1.3. Búsqueda de la bibliografía y de las fuentes

D.1.4. Crítica de las fuentes

D.1.5. Elaboración del estudio

D.1.6. Citas y referencias

D.2. Expresión gráfica y exposición oral

D.3. Edición de documentos electrónicos

D.4. Citas y referencias

E. Valores y estudio del Derecho

F. La profesión de jurista

¿Cómo se puede acceder a los contenidos? A través del índice de contenidos que se muestra en la página principal del módulo en Aula Virtual, y también en la página Web (www.uv.es/legalskills) que enlaza con Aula Virtual. Esto permite encontrar la información que se busca de forma sistemática, aunque requiere un conocimiento previo del programa.
Otra posibilidad es a través de un buscador que, en estos momentos, tenemos pendiente de desarrollar, y que permitiría buscar por términos, preguntas...

¿Qué software y medios hemos utilizado para generar contenidos? La generación de los contenidos se ha realizado con el programa eXeLearning ${ }^{8}$ (http://www.exelearning.org http://www.exe-spain.es ). Se trata un editor para generar contenidos docentes en formato XML o HTML y empaquetarlos en formato SCORM 1.2. Este formato es importado a Aula Virtual desde la herramienta Materiales en línea. Con él se permite, entre otras cosas, una visualización sencilla e integrada de textos, enlaces, realización de ejercicios con autocorrección, la visualización de material audiovisual o simulaciones (Figura 3).

La utilización del programa no es compleja, aunque exige formación previa. Una vez generados los contenidos docentes nos ha planteado problemas de estabilidad en algunos navegadores. Podemos decir que exige un conocimiento técnicamente complejo para el profesor y el apoyo de personal informático.

La grabación de pantalla de simulaciones en video se ha realizado con el programa iShowU ${ }^{9}$ (http://www.shinywhitebox.com/). La simulación permite mostrar cómo se hace algo: por ejemplo, búsqueda de información (normas, jurisprudencia...), realización de una actividad (citar correctamente una fuente de información...).

La edición de video se ha realizado con iMovie y QuickTime. Para la conversión de formatos de video se ha utilizado el programa ffmpegx (http://ffmpegx.com), Toast Titanium y también QuickTime. Por último, se ha utilizado el Servidor Multimedia de la Universitat de València (http://mmedia.uv.es) para depositar los archivos de video, realizar la conversión a formato flash o mp4, y permitir la difusión en streaming.

\footnotetext{
9 Las características recomendadas que proporcionan mejor rapidez y calidad en los archivos generados con el capturador de pantalla iShow son las siguientes; “Small, High Quality, Small file”, 30 imágenes/segundo; Compresión codificador: H.264; tamaño captura: $978 \times 684$ pixeles. El formato de salida es .mov, usado por QuickTime Player.
} 


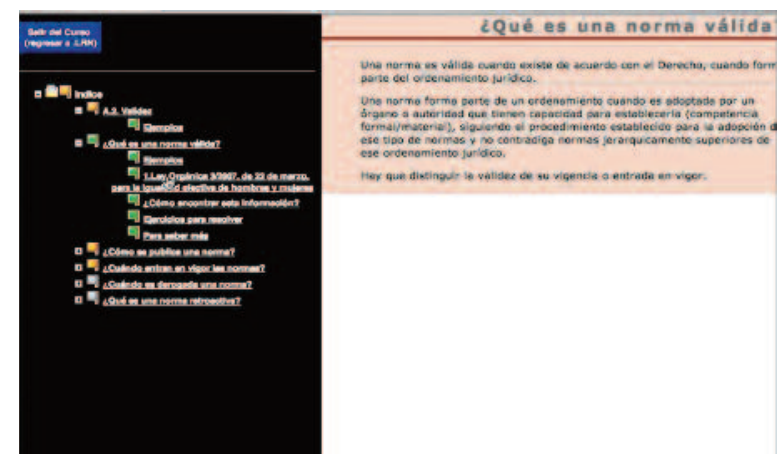

Figura 4. Ejemplo de funcionamiento de los Materiales en línea desde Aula Virtual consultando un contenido

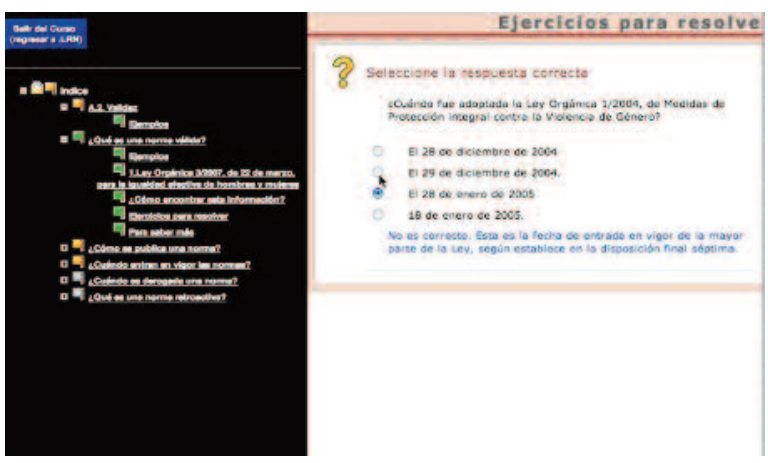

Figura 6. Ejemplo de funcionamiento de un ejercicio de autocorrección en los Materiales en línea desde Aula Virtual

El programa iMovie permite una edición sencilla y profesional de la imagen. Sin embargo, la importación desde el formato .mov y su posterior compresión hace que algunos elementos pierdan calidad, por ejemplo los documentos con texto escrito o letras.

QuicktimePro, aunque es un programa con ciertas limitaciones en lo relativo a la edición de imagen, mantiene la calidad de la grabación de pantalla. Para depositarlo en el Servidor Multimedia se ha guardado como formato .m4v (iPod) que ha mantenido mejor calidad que otros (.avi, Divx).

El Servidor Multimedia de la UV, integrado en Aula Virtual, es sencillo para convertir videos a formato flash (.flv) o pp4, si bien los archivos de video deben ser inferiores a 1GB. El espacio global susceptible de ser utilizado está limitado a 3 GB, aunque en este momento lo hemos ampliado al doble de capacidad. En un principio, se nos planteó la dificultad de depositar archivos de grupos o instituciones, debido a que el proceso de trabajo del servidor estaba y sigue estando vinculado a las cuentas individuales que la universidad ofrece a su personal docente y administrativo y a sus estudiantes, pero esto fue solucionado por el Servei d'Informàtica creando una cuenta de grupo que vinculoó al módulo de Habilidades. Los videos generados en formato flash pueden ser vistos por el público en general o también se puede utilizar la opción de contraseña. En nuestro caso, hemos optado por no utilizar las opciones anteriores, sino otra tercera que limita su visualización a determinados ordenadores que el profesor y los técnicos pueden definir a través de la limitación del uso a unos números IP determinados, de manera que solo puedan verse desde ordenadores de la Universitat de València o en VPN (red privada virtual).

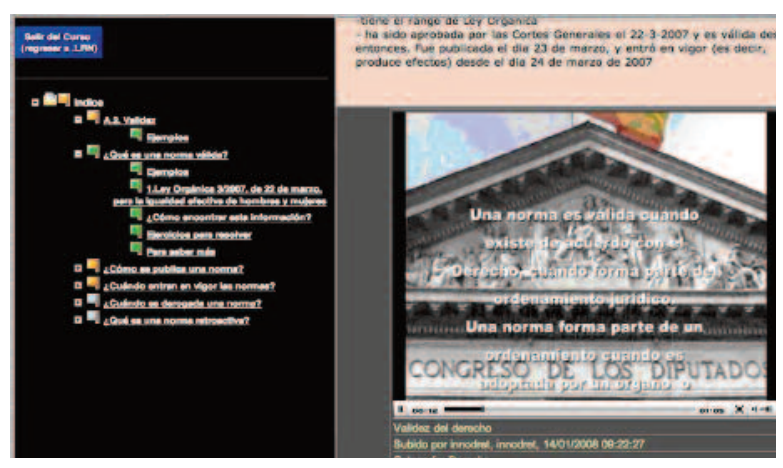

Figura 5. Ejemplo de simulación en los Materiales en línea desde Aula Virtual conectando con un video del servidor multimedia"
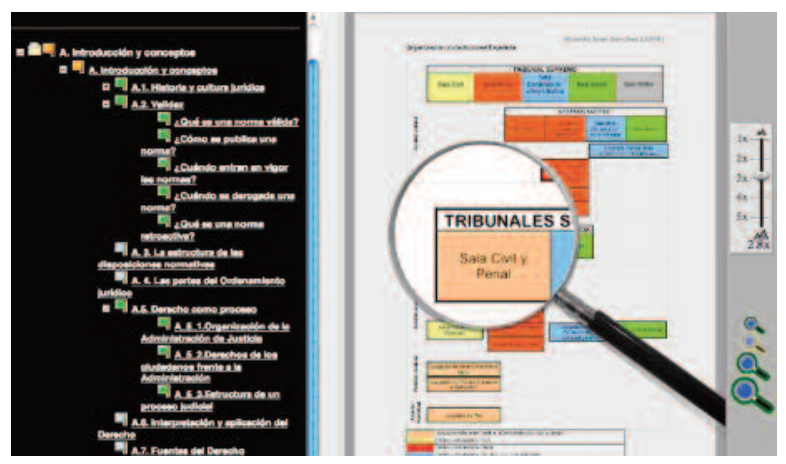

Figura 7. Ejemplo de funcionamiento de herramienta de ampliación en los Materiales en línea desde Aula Virtual

También se han realizado pruebas con un módulo Wiki, xowiki, que es una implementación de wiki para OpenACS en XoTel y que es uno de los módulos/herramientas de la plataforma Aula Virtual. Sin embargo, su uso para la generación y búsqueda de contenidos no ha sido satisfactoria.

Xowiki permite el desarrollo de webs colaborativas y editar, borrar o modificar los contenidos, que siempre se nos muestran en su versión más actualizada. Ésta es su mayor ventaja, que evita con ello la existencia de diversas copias de un mismo documento pues permite trabajar en uno mismo compartido por varios usuarios que lo modifican. No obstante a nuestro modo de ver ofrece demasiadas desventajas. La lógica que funciona en la aplicación es la del enlace constante de las nuevas páginas a las secciones y los comentarios a esos contenidos. De cualquier manera es difícil organizar de forma clara el contenido cuando te enfrentas con la herramienta por primera vez. Es una aplicación poco intuitiva que además requiere de mucho tiempo de investigación hasta familiarizarse con el procedimiento y poder hacer cosas más elaboradas.

\section{Resultados: material educativo obtenido}

Como se ha señalado, parte de los contenidos del programa se pueden encontrar en el módulo Habilidades jurídicas, herramienta de Materiales en línea, en Aula Virtual. Una versión previa de la estructura de contenido se encuentra en http://www.uv.es/legalskills.

Los contenidos educativos generados con eXeLearning se encuentran en proceso de elaboración, y está prevista su puesta en marcha para una primera evaluación a partir de febrero de 2009. Los videos generados y convertidos a formato flash se pueden encontrar en el Servidor Multimedia 
de la Universitat de València. También se ha generado un índice que puede consultarse por criterio cronológico y por materias.

Por ejemplo, el concepto de validez se ha dividido en varias preguntas: ¿qué es una norma válida?, ¿cómo se publica una norma?, ¿cuándo entran en vigor las normas?, ¿̇cuándo se deroga una norma?, ¿qué es una norma retroactiva?.. Todas estas cuestiones tratan de responder a este aspecto. Para ello, cada apartado, -por ejemplo, “¿qué es una norma válida?” que se refiere a cuándo se adoptan las normas- se subdivide en un apartado de contenido, una simulación con ejemplos, ejercicios para resolver, y Para saber más.

En el apartado de contenido, de forma sintética, se muestra una definición o concepto, en este caso sobre el concepto de validez. La finalidad es que con una rápida lectura uno pueda recordar o hacerse una idea del concepto (Figura 4). En el apartado de la simulación se muestra con un ejemplo de cuándo ha sido aprobada una ley. Por ejemplo, la Ley Orgánica 3/2007, de 22 de marzo para la igualdad efectiva de hombres y mujeres, aparece en un video en el que se muestra cómo encontrarla en el BOE, y en una base de datos dónde se puede encontrar esta información (Figura 5).

En el apartado de Ejercicios para resolver (Figura 6), se puede practicar lo aprendido y, para ello, se pregunta alguna cuestión relacionada con los conocimientos que se han presentado anteriormente. Las respuestas se autocorrigen y permiten retroalimentación con una información determinada, según la respuesta sea correcta o incorrecta. Por ejemplo, se pregunta ¿̇cuándo fue adoptada la Ley Orgánica 1/2004, de Medidas de Protección Integral contra la Violencia de Género? Para responder a la pregunta hay una pista y cuatro opciones. Con ello, además de clarificar el concepto de adopción o aprobación de una norma, se compara con otros: el de publicación, entrada en vigor, vigencia.... Por último, el apartado Para saber más, remite de forma sintética a otras fuentes de información que permiten ampliar y profundizar en ese asunto.

Esta herramienta permite realizar otra serie de actividades o funciones: lecturas, casos, preguntas verdadero-falso... y también ampliar imágenes (Figura 7).

\section{Autoevaluación y conclusiones}

En relación al programa de contenidos previsto en un comienzo tan solo se han desarrollado e implantado algunos aspectos, debido a tres cuestiones:

1) las dificultades previas de elección de una plataforma y una herramienta para mostrar los contenidos;

2) la necesidad de formación requerida por las aplicaciones informáticas utilizadas; y

3) el abandono de profesores que se habían comprometido en un principio.

Por tanto, podríamos decir que nos encontramos aún al principio en algunos aspectos y hemos avanzado en otros. Podemos señalar las dificultades y obstáculos que para el futuro.

Desde el punto de vista del personal habría que destacar la exigencia de mayor implicación por parte de los profesores. Además, y aunque en un principio nos planteamos la necesidad de personal técnico y la colaboración con el Servei d'Audiovisuals, la dinámica de trabajo nos llevó a decidir que era más eficaz la colaboración de personal en formación. De hecho, en una fase avanzada se optó por realizar una convocatoria de dos becas de colaboración. En el futuro parece necesario seguir contando con la colaboración de becarios en formación o personal técnico que auxilie en la elabora- ción de contenidos a los profesores. También con la colaboración de más profesores de diversas disciplinas.

En relación a los contenidos parece necesario trabajar en su simplificación y adecuación al medio que se presenta. Por tanto, desde el punto de vista de los contenidos falta: a) mejorar la coordinación de contenidos de la plataforma; b) hacerlos accesibles y adecuados a la finalidad que se pretende.

En relación a las dificultades técnicas cabría señalar los problemas que se han detectado con formatos de imagen, empaquetado, la plataforma, la compatibilidad con los exploradores, y la estabilidad de las aplicaciones. Esto exige también una mayor formación en las técnicas utilizadas. Por tanto, desde el punto de vista técnico falta: a) incluir un buscador de contenidos; b) posibilitar que los contenidos del módulo estén disponibles para todos los alumnos y profesores cada curso; c) sistematizar un catálogo de materiales audiovisuales para uso docente e investigador; d) la estabilidad de las aplicaciones utilizadas

En relación a la búsqueda de contenidos hay que señalar que esto es una limitación de los contenidos generados en SCORM y tampoco ha sido fructífera la utilización de la herramienta Xowiki. Ésta es una de las cuestiones en las que se está trabajando en la actualidad.

\section{Bibliografía}

ACLEC, (1996). Lord Chancellor's Advisory Committee on Legal Education and Conduct, First Report on Legal Education and Training London 1996 http://www.ukcle.ac.uk/resources/aclec/index.html

ANECA(2006). Libro Blanco. Título de Grado en Derecho, Madrid, Agencia Nacional de Evaluación de la Calidad y Acreditación. http://www.aneca.es/activin/docs/libroblanco derecho def.pdf

Dasí Coscollar, Angels.; García Añón, José; Huguet Roig, Ana; Juan Sánchez, Ricardo; Montagud Mascarell, M.Dolores; Rollnert Liern, Göran (2007). Innovación educativa en la Universidad: ADE-Derecho. Valencia: Publicacions de la Universitat de Valencia. http://www.uv.es/oce/web\%20castellano/ADE-Derecho.pdf)

Delgado, Ana M. ${ }^{\text {a }}$ Oliver, Rafael (2003). Enseñanza del Derecho y tecnologías de la información y la comunicación http://www.uoc.edu/dt/20310/index.html

Delgado García, Ana María (Coord.) (2005). Competencia y diseño de la evaluación continua y final en el Espacio Europeo de Educación Superior, Programa de Estudios y Análisis EZ2005-054. Madrid: Dirección General de Universidades, Ministerio de Educación y Ciencia 2005 http://www.mec.es/univ/proyectos2005/EA20050054.pdf

García Martínez, Jesús (2007). Las competencias y la nueva organización de la enseñanza. En María Reyes León Benítez (coord.), La licenciatura en Derecho en el contexto de la Convergencia Europea. Valencia: Tirant lo Blanch, pp. 163-177.

Gold, N.; Mackie, K.; Twining, W. (eds.) (1988). Learning Lawyers' Skills. London: Butterworths.

González, Julia y Robert Wagenaar (eds.) (2003). Tuning Educational Structures in Europe. Informe final Proyecto Piloto-Fase 1, 2002; Bilbao, Universidad de Deusto y Universidad de Groningen.http://www.relint.deusto.es/TUNINGProject/spanish/doc2 fase1.asp 
León Benítez, María Reyes (2007). La enseñanza del Derecho basada en competencias. En María Reyes León Benítez (coord.), La licenciatura en Derecho en el contexto de la Convergencia Europea.Valencia: Tirant lo Blanch, pp.179-197

Maughan, Caroline y Webb, Julian (1995). Lawyering Skills and the Legal Process. Cambridge: Cambridge University Press, 2005.

Miguel Díaz, Mario de (Coord.); (2006). Metodologías de enseñanza y aprendizaje para el desarrollo de competencias, Madrid: Alianza.

Pérez Lledó, Juan Antonio (2002). Teoría y práctica de la enseñanza del Derecho. Anuario de la Facultad de Derecho de la Universidad Autónoma de Madrid, 6 , pp.197-268.

Webb, J. y Maughan, C. (eds.) (1996). Teaching Lawyers' Skills, London: Butterworths.

Williams, Gleanville. (1945). Learning the Law, A.T.H. (ed.) Smith, London: Sweet\&Maxwell, 2002.

\section{| Cita recomendada de este artículo}

García Añón, José et al. (2008). Diseño de materiales para el aprendizaje de habilidades jurídicas fundamentales. @tic. revista d'innovació educativa. ( $\left.n^{\circ} 1\right)$ http://ojs.uv.es/index.php/attic/article/view/49/44 Fecha de consulta, dd/mm/aa 